EVIDENCE BASED PUBLIC HEALTH POLICY AND PRACTICE

\title{
Ethical issues in public health: a qualitative study of public health practice in Scotland
}

\section{W A Rogers}

J Epidemiol Community Health 2004;58:446-450. doi: 10.1136/jech.2003.013417

\section{Correspondence to: Associate Profressor W A Rogers, Department of Medical Education, Flinders University, GPO Box 2100, Adelaide SA 5001, Australia; wendy. rogers@flinders.edu.au}

Accepted for publication 3 September 2003 ethical issues encountered by staff in the development and implementation of Study objective: To identify ethical issues enco
public health activities at two sites in Scotland. Design: Qualitative research study involving face to face semi-structured interviews with participants. Setting: A public health directorate in a National Health Service Trust, and a public health demonstration project in child health.

Participants: Health promotion specialists, managers, nurses, public health consultants and specialists, researchers, trainees, and other public health staff.

Main results: Three main categories of ethical issues were identified: paternalism, responsibilities, and ethical decision making. Consulting with the community and sharing information raised issues of paternalism and honesty. Participants identified multiple and sometimes conflicting responsibilities. Barriers to fulfilling responsibilities included meeting targets, working with partners, and political influences. Defining the limits of responsibilities posed challenges. Participants identified values for ideal decision making, but lack of time often led to a more pragmatic approach.

Conclusion: These empirical findings complement and extend existing discussions of public health ethics, emphasising the complex nature of ethical issues in public health. The implications for public health policy and future research are discussed.
$P$ ublic health is concerned with preventing disease, prolonging life, and promoting health. The value of good health and the responsibilities of governments to safeguard the health of their citizens are widely accepted. However, the actions required to protect the public health raise various ethical issues. ${ }^{1}$ There are tensions between achieving benefits for whole populations and protecting individuals' rights. ${ }^{2}{ }^{3}$ Epidemiological data collection can conflict with the right to privacy. Health promotion interventions may be paternalistic or intrusive. ${ }^{4}$ Resources for public health compete with other legitimate demands. ${ }^{5}$

There is increasing interest in public health ethics, ${ }^{6-8}$ with contributions from virtue ethics, ${ }^{9}$ communitarian, ${ }^{10}{ }^{11}$ and rights based accounts. ${ }^{12}{ }^{13}$ These necessary conceptual analyses may be complemented and strengthened by empirical research, leading to a richer understanding of moral issues. ${ }^{14}$

This study aimed to identify ethical issues encountered by public health professionals engaged in service delivery at two Scottish sites.

\section{METHODS}

\section{Permissions and ethics approval}

Ethical approval was granted by the Lothian Primary Care/ Public and Mental Health and the Greater Glasgow Primary Care-Community and Mental Health Research Ethics Committees.

\section{Participants}

The sampling strategy aimed to access a wide range of roles and occupations, as diversity of professional role is potentially significant in public health ethics. ${ }^{1}$ Lists of staff were obtained from the Public Health Directorate of the Lothian NHS Board and the Glasgow Starting Well Demonstration Project in child health. These were used to generate a sampling frame, with each cell representing a different role or profession (see box 1). There were 25 cells in the Lothian frame and six in the Glasgow frame. In cells with only one person (for example, a unique position), that person was selected. Random selection was used in cells with more than one person. In cells with over 10 people (health promotion specialists and health visitors), up to four people were recruited.

Potential recruits were sent a letter, with telephone follow up one week later. Recruitment letters were sent out in two rounds; the second round targeted cells with no recruit to date. Thirty four letters were sent out in Lothian, leading to 24 interviews. Fifteen letters were sent out in Glasgow, leading to nine interviews. The overall response rate was $67 \%$. Recruiting stopped once I had requested at least one interview from every cell in the sampling frame.

\section{Qualitative interviews}

Interviews took place in late 2002, either at the work place of the interviewee or another place of their choice. All participants gave written informed consent to be interviewed. Interviews lasted between 35 and 90 minutes; most were about one hour. The interview covered four main topic areas which were explored with open-ended and clarifying questions (see box 2).

Participants' responses were recorded in contemporaneous notes taken by the author, with additional notes written immediately afterwards. Material from each interview was entered onto computer within 24 hours, and checked against the original interview notes, generating a dataset of 33 texts.

\section{Analysis}

Analysis involved coding the data into categories. ${ }^{15}$ Initial descriptive codes were derived from the data and explored in later interviews. Analysis continued after the completion of data collection. Coded material was compared and organised into themes that were then grouped into three central categories reflecting the major issues described by participants. Two of these categories, "responsibilities" and "ethical decision making", derive solely from the data. Material 
Box 1 Examples of professional groups included in the sampling frame (potentially identifying roles have been omitted)

- Health promotion specialists

- Managers

- Nurses

- Project staff

- Public health consultants

- Public health specialists

- Researchers

- Trainees in public health (medical and non-medical)

initially coded as community consultation/consent and information issues was grouped into the "paternalism" category as this material fitted the theoretical concept of paternalism. Material not included in the central categories was retained as separate themes. To strengthen validity, the research findings were fed back to participants who attended presentations offered at three different venues. ${ }^{16}$

\section{RESULTS}

\section{Paternalism}

Public health staff described two situations involving paternalism: negotiating community consultation and/or consent, and withholding information from the public.

Participants expressed the view that community consultation can be paternalistic. Consultation was required in some public health contexts, but the power or control that communities could actually exert over defining their needs or choosing interventions was limited by professionally imposed parameters, such as a requirement for an evidence base. Participants observed that more deprived populations, who are most likely to have interventions imposed, are least likely to participate in consultation.

There was no mechanism for obtaining informed consent from communities for new interventions, such as the Starting Well programme of intensive home visiting for young children, nor for people to opt out of community wide interventions.

Decisions about sharing information with the public raised questions of paternalism in two situations. The commonest view point of staff developing information leaflets was that information should be limited, in part to avoid causing the public fear and anxiety. However, some participants argued that people want more rather than less information.

Participants described the difficulty of deciding how open to be with the public about errors or accidents, and how to be honest without causing public anxiety. One strategy was to release intentionally opaque information, through careful wording. People dealing with current incidents had to take account of sometimes inadequate existing levels of public information.

On the other hand, participants recognised that the public demanded high levels of honesty, and that suspicion of cover ups could be equally harmful to public trust. Participants described the challenges raised by their desire for honesty, the difficulty of getting a fair hearing in the media, the complexity of public health information, and the generally poor understanding of public health among politicians, the public, and the media.

\section{Responsibilities}

Participants described multiple and sometimes conflicting responsibilities to different stakeholders (see box 3). Most

\section{Box 2 Topic areas included in interview}

- Nature of work

- Ethical issues encountered in work

- Responsibilities

- Ethics education or training

participants identified a primary responsibility towards the public, to protect and promote community health, and to further population health interests. Many participants (consultants, researchers, nurses, managers) identified day to day responsibilities towards the NHS Board or project management team. In turn the Board was seen as responsible to the Scottish Executive Health Department and ultimately to the public. Some participants (managers and consultants) described a responsibility towards NHS staff in terms of ensuring that their decisions supported those providing clinical services. Some participants (researchers, managers, consultants) identified a responsibility towards the Scottish Parliament, and through them to the electorate. Some participants (doctors, nurses, and health promotion specialists) described a responsibility towards professional and personal values. Of these, some thought that personal values should not intrude into professional ethics, but for others, their personal values shaped the boundaries within which they could discharge their work responsibilities.

For many public health staff there was no direct relationship with the public as public health work often entails dealing with unidentified individuals. The community was thought to be largely unaware of public health activities, and unlikely to offer any positive feedback, with publicity usually limited to adverse events such as disease outbreaks. Some participants said that the absence of a direct relationship made it more difficult to feel responsible to the public. Others said that the lack of a relationship could be an advantage in fulfilling some responsibilities, such as resource allocation decisions. This was explained in terms of decisions that are good at a community level are often decisions that one would neither want for oneself, nor want to deliver as clinicians, for example limiting access to expensive therapies.

Two occupational groups described ethical issues around setting the boundaries of responsibility. Starting Well staff involved in home visiting (health visitors and nursery nurses) had specific responsibilities to deliver the agreed package of care, and were aware of the importance of adhering to protocols. But at times, the agreed package of care did not accord with the immediate needs of particular families. In these situations, responsibility to the child was felt to be in conflict with responsibility to the project.

The other group who questioned the limits of their responsibilities were involved in delivering care for specific population groups. The issue here was how much of a champion to be for one's area of responsibility, given that

\section{Box 3 Responsibilities identified by participants}

- To the population

- To the NHS Board

- To NHS staff

- To elected representatives

- To uphold professional and personal values 
intensive lobbying may be necessary to secure resources. Some accepted the role of champion and described intensive lobbying for their cause. For others, this behaviour conflicted with their own personal style and/or view of appropriate professional behaviour.

Participants described barriers to fulfilling their responsibilities, related to targets, working with partners, and political influences. Meeting targets for accountability purposes raised a number of ethical issues. These included inequitable service delivery, and compromised patient autonomy. Meeting targets was sometimes beyond the power or capability of staff and diverted resources away from other equally important health priorities. Participants described ways that targets can mislead and reduce complex issues to simple, measurable solutions.

Participants described issues related to working with partners. At times lack of shared goals and commitment led to misunderstandings and potential exploitation. Combining different professional groups presented challenges such as differing values and work ethos, competition between groups, and diverse professional standards.

Many participants mentioned the close relation between public health and politics. Responding to queries from politicians diverted time and energy away from responsibilities that were often felt to be more important. Some participants felt that members of parliament used their influence to assist patients who were unhappy with the system, but this was unfair to similar patients who waited patiently. Political attention made it particularly difficult to withdraw a service, even if this decision was justifiable in terms of resource allocation priorities. On the other hand, political attention could sometimes be beneficial, drawing publicity and resources towards a topic that might otherwise be a low priority. Politicians were perceived to be motivated by self interest in contrast with public health staff who described themselves as genuinely working for the good of the community.

\section{Ethical decision making}

Despite a desire for evidence based decision making, participants described a range of values that influenced the process, although these influences were not always overtly recognised. Participants identified a number of ideal values that should characterise the decision making process (see box 4).

Making decisions based upon these values was described as intrinsically right or good, reflecting core values of public health. In addition, two instrumental benefits were identified: decisions made like this were more likely to be agreed by all parties and be successfully implemented; and this process shared responsibility, which was seen as appropriate by some participants.

Box 4 Values for ideal decision making in public health

- Open, honest, transparent

- Democratic, consensual

- Inclusive, consultative

- Rational, deliberative, systematic

- Evidence based

- Weighing costs and benefits, affordable

- Respectful

- Mindful of equality
Participants identified various ways in which these ideals were compromised. Lack of time was an important factor, limiting consultation processes, or searches for evidence and leading to loss of sometimes significant detail. Decision making often took place with a sense of urgency (in response to events or political pressure) and this was described as compromising the process.

"Pragmatic" was a term used by several participants to characterise the decision making process, in recognition of the multiple pressures and sometimes incompatible aims involved. Pragmatism involved asking "What will be good enough, or achievable?" and finding the path of least resistance.

Participants described complexities around the processes of decision making and fulfilling responsibilities. Some decisions required political approval, leading to tensions between NHS Boards' responsibility for healthcare delivery and their political accountability. There was the perception that politicians did not want to be associated with potentially unpopular decisions, leaving a decisional vacuum at times.

Three additional themes are presented here: relations with pharmaceutical companies, confidentiality, and education.

Relations with pharmaceutical companies raised a number of ethical issues. Participants described the potential benefits of accepting industry funding for services or resources that would otherwise not be available, for example equipment or nurses' salaries. This benefit was balanced against perceived problems including:

- inequitable distribution of services provided by pharmaceutical companies;

- circumventing usual prioritising processes by providing short term funding for services that then had to be continued with general funds;

- pressure to accept drugs with a poor evidence base;

- the inappropriateness of commercial funding in a national health service;

- lobbying methods used by pharmaceutical companies; and

- lack of an agreed mechanism for identifying and/or working with staff who had potential conflicts of interest.

Participants described a lack of policy or guidelines regarding relations with pharmaceutical companies.

Confidentiality was described as an ethical issue by most participants. Most were aware of the importance of maintaining confidentiality and felt comfortable with the procedures in place to do this. Pressures on confidentiality arose from partners who might lack awareness or adequate systems to maintain confidentiality, and some research and evaluation activities. At times, public health activities were hindered by clinicians maintaining patient confidentiality.

Very few participants could recall ethics education as part of their public health training. Some participants (nurses, doctors, health promotion specialists) described ethics as part of their original professional training. A small group

\section{Key points}

- Public health professionals operate in an ethically complex environment.

- Ethical issues identified by participants include paternalism and honesty, conflicting responsibilities, pressures on decision making, and uncomfortable relations with the pharmaceutical industry. 
identified formal opportunities for ethics discussion in the workplace, but most relied upon informal discussions.

\section{DISCUSSION}

This original study systematically recorded ethical issues identified by public health professionals, thereby complementing and extending the existing literature.

Issues of community consent have been raised by other authors. ${ }^{26}$ One suggestion has been to focus upon avoiding exploitation, rather than trying to gain individual informed consent for community wide interventions. ${ }^{17}$ Improving community consultation processes through greater clarity and openness may be of benefit in avoiding paternalism.

Competing values and obligations are central issues in public health ethics. ${ }^{2}$ This study supports that view; multiple and sometimes conflicting responsibilities were an important issue. How should public health professionals respond, especially when there is conflict between responsibilities to the public and to their employer?

The claim that politics is a necessary part of public health is supported by this study. ${ }^{1}$ The limits of political interference in the day to day workings of public health deserve careful debate.

While not using the explicit language of virtue ethics, participants identified the difficulties of maintaining public trust, and being honest. A focus on the virtues and professional conduct has been suggested as an important part of public health ethics. ${ }^{18}$ We need to discover ways of supporting virtuous practice, such as opportunities for reflective discussions, and mentoring.

Ethical decision making presents complex challenges. The "ideal" decision making values described in this study are similar to those in a framework for ethical governance developed by the Highland NHS Board ${ }^{19}$ (see box 5). The importance of process, as well as the outcomes of decisions, indicates that utilitarianism alone is unlikely to provide an adequate account of public health ethics, and that other considerations are necessary. ${ }^{6}$

Relations between the pharmaceutical industry and public health deserve greater attention. Despite the topicality of this

\section{Policy implications}

- Debate about the responsibilities of the public health workforce would help to clarify issues identified here. This could be facilitated by NHS Boards or professional bodies. Robust community involvement through citizens' juries or panels would help to build understanding of and trust in the workings of public health.

- Specific policy on relations with the pharmaceutical industry would provide support to public health consultants. Policy could be developed at the level of individual NHS Boards, or nationally by the Faculty of Public Health of the Royal College of Physicians.

- Strengthening ethics education in public health training requires the development of expertise, curriculums, and resources. These initiatives could be led by universities offering postgraduate courses in public health, or by professional bodies representing sectors of the public health workforce.

- Implementing these changes would raise the profile of public health ethics and afford opportunities for discussions. The costs are comparatively modest and significant changes are achievable within a two to five year time frame.
Box 5 NHS Highland principles for ethical

decision making

- Accountability

- Justice

- Quality

- Realism

- Engagement

- Flexibility

- Meaningful relationships

issue, ${ }^{20}$ there is little guidance for public health consultants. This study suggests that specific policy may be one way of addressing this issue.

The findings about ethics education and training concur with those of Kessel who found that public health ethics teaching to medical undergraduates and public health postgraduates is patchy and often minimal. ${ }^{21}$ Exploring professional differences and role specific responsibilities will help to inform educational initiatives.

This study has some limitations. Because of concerns about confidentiality, interviews were not taped, and it is possible that important material was omitted in the note taking process. The sampling strategy aimed for breadth of professional roles; this meant that differences between professional groups (such as nurses, health promotion specialists, and doctors), could not be explored in depth. Analysis was performed by the author alone so that this necessarily reflects her own preconceptions about public health ethics. Generalisability may be limited because of these factors. However, presentation of results to relevant public health audiences (including study participants) has met with endorsement as to the nature and importance of the problems identified, giving some confidence that these issues are relevant to the wider public health community.

The importance of this work lies in building links between ethical analysis and the practical work of public health. These findings are relevant to further theoretical and empirical research, and to applied endeavours such as developing public health ethics curriculums and policies. Action on all of these fronts will move us towards greater appreciation of the ethical challenges of public health and how best to meet them.

\section{ACKNOWLEDGEMENTS}

I would like to thank the participants of this study and the public health community in Scotland who were generous with their time and interest.

Funding: This study was funded by a visiting fellowship in public health from the Chief Scientist Office of the Scottish Executive Health Department, hosted by the Department of Community Health Sciences at the University of Edinburgh. The author also received support from the Australian National Health and Medical Research Council (fellowship ID 007129)

Conflicts of interest: none declared.

\section{REFERENCES}

1 Callahan D, Jennings B. Ethics and public health: forging a strong relationship. Am J Public Health 2002;92:169-76.

2 Weed DL, McKeown RE. Science, ethics, and professional public health practice. J Epidemiol Community Health 2003;57:4-5.

3 Gostin L. Public health law in a new century. Part II: public health powers and limits. JAMA 2000;283:2979-84.

4 Downie RS, Fyfe C, Tannahill A. Health promotion: models and values. Oxford: Oxford University Press, 1990.

5 Michaelis A. Priority-setting ethics in public health. J Public Health Policy 2002;23:399-412. 
6 Roberts M, Reich M. Ethical analysis in public health. Lancet 2002;359:1055-9.

7 Kass N. An ethics framework for public health. Am J Public Health 2001;91:1776-82.

8 Mullan F. Don Quixote, Machiavelli and Robin Hood: public health practice past, present and future. Am J Public Health 2000;90:702-7.

9 Horner JS. For debate: the virtuous public health physician. $J$ Public Health Med 2000;22:48-53

10 Beauchamp D. Community: The neglected tradition of public health. In: Beauchamp D, Steinbock B, eds. New ethics for the public's health. New York: Oxford University Press, 1999.

11 Mooney G. "Communitarian claims" as an ethical basis for allocating health care resources. Soc Sci Med 1998:47:1 171-80.

12 Mann JM. Medicine and public health, ethics and human rights. Hastings Cent Rep 1997;27:6-13.

13 Rodriguez-Garcia R, Akhter M. Human rights: the foundation of public health practice. Am J Public Health 2000;90:693-4.
14 Sulmasy D, Sugarman J. The many methods of medical ethics. In: Sugarman J, Sulmasy D, eds. Methods in medical ethics. Washington: Georgetown University Press, 2001.

15 Pope C, Ziebland S, Mays N. Qualitative research in health care. BMJ 2000;320:114-16.

16 Malterud K. Qualitative research: standards, challenges, and guidelines. Lancet $2001 ; 358: 483-8$.

17 Kassell J, Young A. Why we should not seek individual informed consent for participation in health services research. J Med Ethics 2002;28:313-17.

18 Weed D, McKeown R. Epidemiology and virtue ethics. Int J Epidemiol 1998;27:343-9.

19 Highland NHS Board. Ethical decision making: a framework for ethical governance. Inverness: Highland NHS Board, 2002.

20 Multiple authors. Series of articles on relationships with the pharmaceutical industry. BMJ 2003;326:1 155-210.

21 Kessel A. Public health ethics: teaching survey and critical review. Soc Sci Med 2003;56: 1439-45.

\section{SPEAKER'S CORNER}

\section{Public health practitioners can learn from the weather forecasters}

n Canada there is a Weather Network channel on the television that provides weather related information 24 hours a day, 7 days a week. This information is also available on the web site. ${ }^{1}$ I hope there will be a television channel for disseminating public health information to the general public in a similar way.

Firstly, public health practitioners can design universal non-word symbols to denote public health events. This is similar to, for example, the picture of a sun, a sun partly covered by clouds, or clouds with snow flakes, which can be easily understood by people speaking different languages.

Secondly, we can provide public health short range and long range forecasts on a number of important indicators. For example, on the weather channel there are local forecasts for this afternoon (temperature, sunny/cloudy, wind, pressure, relative humidity); conditions this evening, overnight and morning; seven day outlook; and long range forecast.

Thirdly, we can use colour coded maps to denote public health situations and predictions in space and time. For example the Weather Network uses the following colour codes for warnings: heavy snow fall (grey), blizzard (white), blowing snow (light green), wind (yellow), freezing rain (light red), heavy rainfall (green), winter storm (red), wind chill (blue).

Fourthly, regular broadcast can be interrupted by "Public Health Alert". For example, "Meteorological Alert" is a severe weather bulletin issued by Environment Canada, with warnings on a bright red background.

Fifthly, information that is relevant and used by the public can be disseminated. Information dissemination should be "user pull" rather than "provider push". For example, the following information is presented in winter on the Weather Network: highway conditions (amount of rain and snow), ski conditions (number of runs open, and snow depth), sunshine getaways (weather conditions in warm resort areas).
Sixthly, information should be presented in an interesting way. For example, today's Weather News is "St John's, Newfoundland, is expected to have $40 \mathrm{~cm}$ of snow this evening." Weather Facts is "it was on this day 35 years ago ...". Weather Quiz is "the coldest capital yesterday was(A) Fredericton NB, (B) Toronto ON, (C) Winnipeg MN, (D) Edmonton $A B$ ?" The answer: "(A) -23 degrees celsius, (B) -20 , (C) -36 , (D) $-20^{\prime \prime}$.

Seventhly, information should be translated to the level understood by the public. For example, a low pressure system is interpreted by the broadcaster "in other words, a weather bomb", wind chill is explained by "with the wind it feels like", and it is further explained "wind chills of -40 to -50 degrees celsius means exposed skin freezes in less than 10 minutes".

Eighthly, education and training can be provided for the public. For example, today's Weather Wise teaches about "what is an isobar?", "how does a snowflake form?", and "why does the Alberta Clipper dump a lot of snow on the East Coast of Canada?"

The basic question before putting in place a public health channel is, how can we sufficiently market public health to the general public so that people will be more concerned about their own health tomorrow than whether it is going to rain or snow tomorrow?

B C K Choi

Population and Public Health Branch, Health Canada, AL no 6701A, 120 Colonnade Road, Ottawa, Ontario K1A 1B4, Canada; Department of Public Health Sciences, University of Toronto; Department of Epidemiology and Community Medicine, University of Ottawa, Canada: Bernard_Choi@hc-sc.gc.co doi: 10.1136/jech.2004.020404

\section{Reference}

1 The Weather Network. http://www.theweathernetwork.com/ (accessed on 26 Jan 2004). 